\title{
Implementando Una Gestión Pública En La Unidad De Gestión Educativa Local De Castrovirreyna
}

\author{
Riveros Poma, Rómulo \\ Unidad de Gestión Educativa Local de Castrovirreyna \\ romulorp6@gmail.com \\ https://orcid.org/0000-0002-1742-7305
}

\section{Resumen:}

En el Sector Educación, durante la crisis sanitaria mundial del COVID 19, los servidores estamos experimentando cambios muy significativos, en la atención al ciudadano, especialmente, solucionar los problemas de falta de conectividad de los estudiantes de EBR, EBA y ETP.

Al sistematizar la primera información de directores de Instituciones Educativas, se demostraron que el $46 \%$ de estudiantes se encontraban sin conectividad a los medios de comunicación y tecnológicos. Resultado preocupante, durante el aislamiento social, que ha motivado a promover diversas alternativas, de cierre de brechas de falta de conectividad, para que los estudiantes reciban las sesiones de aprendizaje, a través de la estrategia del Ministerio de Educación de APRENDO EN CASA.

Pero, las actividades administrativas que cumplimos estaban instauradas con el "cumplimiento de funciones". Un problema crucial que no permitía movilizar propuestas, por no estar acorde a las acciones establecidas de costumbre, hasta que estalló en cambio de responsables de algunas áreas, para sensibilizar adecuadamente a trabajadores para emprender nuevas intervenciones durante el trabajo remoto.

Desde ahí, las autoridades educativas promueven el cambio e inician la toma de decisiones colegiadas y coordinaciones interinstitucionales con autoridades municipales, políticas, de salud y policiales de la provincia de Castrovirreyna, con la finalidad de cerrar brechas de falta de conectividad de los estudiantes. Esta iniciativa tuvo buena aceptación y se emprendió arribar acuerdos e iniciación de implementación de LAS GUÍAS DE APRENDIZAJE, que son manuales por área curricular para que el estudiante desarrolle con mayor facilidad el contenido de los textos de lectura de cada grado. Además, se instalaron con 8 estaciones de internet satelital y un radio emisora en la infraestructura de la UGEL Castrovirreyna.

Entonces, el nacimiento de la gestión pública propiamente dicha, en la UGEL Castrovirreyna, nace como efecto de la crisis sanitaria del COVID 19, donde hay participación voluntaria de padres de familia, autoridades comunales y autoridades locales, las que están orientadas a enfatizar el rol de los ciudadanos en la formulación de las políticas públicas y la coproducción de servicios: enmarcado en la transparencia y lucha contra la corrupción.

Palabras clave: Gestión pública; Crisis sanitaria; Cambio

Implementando una gobernanza pública en la UGEL de Castrovirreyna - Riveros Poma,

Rómulo

DOI: https://doi.org/10.18800/cige2020.005 
Congreso Internacional de Gestión Educativa, 06 y 07 de noviembre de 2020.

Pontificia Universidad Católica del Perú

\begin{abstract}
:
In the Education Sector, during the global health crisis of COVID 19, the servers are experiencing very significant changes, in the attention to the citizen, especially, solving the problems of lack of connectivity of the EBR, EBA and ETP students.
\end{abstract}

When systematizing the first information from directors of Educational Institutions, it was shown that $46 \%$ of students were without connectivity to the media and technology. Worrying result, during social isolation, which has motivated the promotion of various alternatives, closing gaps of lack of connectivity, so that students receive learning sessions, through the strategy of the Ministry of Education of LEARNING AT HOME.

But, the administrative activities that we carried out were established with the "fulfillment of functions." A crucial problem that did not allow the mobilization of proposals, as it was not in accordance with the usual established actions, until it broke out instead of those responsible for some areas, to adequately sensitize workers to undertake new interventions during remote work.

From there, the educational authorities promote change and initiate collegiate decisionmaking and inter-institutional coordination with municipal, political, health and police authorities in the province of Castrovirreyna, in order to close gaps in the lack of connectivity of students. This initiative was well accepted and agreements and initiation of implementation of THE LEARNING GUIDES were undertaken, which are manuals by curricular area so that the student can more easily develop the content of the reading texts for each grade. In addition, 8 satellite internet stations and a radio station were installed in the UGEL Castrovirreyna infrastructure.

Then, the birth of public management itself, in the UGEL Castrovirreyna, was born as a result of the health crisis of COVID 19, where there is voluntary participation of parents, community authorities and local authorities, which are oriented to emphasize the role of citizens in the formulation of public policies and the co-production of services: framed in transparency and the fight against corruption.

Keywords: Public management; Health crisis; Change

\title{
Introducción.
}

El acceso al mejor estándar accesible de educación es un derecho humano fundamental y un imperativo moral para el desarrollo sostenible de los pueblos. Una educación de calidad y equitativa tiene el propósito de atender al estudiante en el mejoramiento de sus aprendizajes, sin excluir por su origen, religión o situación de pobreza, implementada por una UGEL, es transformar las condiciones sociales existentes hacia lo colectivamente deseable, mejor calidad de vida, innovadora, emprendedora y sostenible.

Pero, durante la crisis mundial del COVID 19, a través del análisis documental, informes de directores de IIEE sobre la estrategia APRENDO EN CASA y visitas domiciliarias, se constató que el centralismo limeño, hizo que las comunidades campesinas de la provincia de Castrovirreyna - Huancavelica, no tengan los servicios básicos de agua, desagüe y energía eléctrica. Y, para la educación a distancia, falta del $46 \%$ de conectividad de radio, televisión e internet, manteniéndose vigentes políticas

Implementando una gobernanza pública en la UGEL de Castrovirreyna - Riveros Poma, Rómulo

DOI: https://doi.org/10.18800/cige2020.005 
Congreso Internacional de Gestión Educativa, 06 y 07 de noviembre de 2020.

Pontificia Universidad Católica del Perú

educativas despectivas de "personajes de gobiernos de turno", especialmente hacia los pobladores de provincias, distritos y contextos rurales.

Es más, algunos servidores del área administrativa de la UGEL Castrovirreyna, solamente "cumplen funciones", pero durante la crisis sanitaria se dificultaron en continuar con lo cotidiano y, por necesidad, optaron a realizar actividades visibles y con resultados, previo monitoreo y acompañamiento de cada Jefe de Línea.

Einstein decía que; "Quien atribuye a la crisis sus fracasos y penuria, violenta su propio talento y respeta más a los problemas que a las soluciones. La verdadera crisis, es la crisis de la incompetencia. El inconveniente de las personas y los países es la pereza para encontrar las salidas y soluciones. Sin crisis no hay desafíos, sin desafíos la vida es una rutina, una lenta agonía. Sin crisis no hay méritos. Es en la crisis donde aflora lo mejor de cada uno, porque sin crisis todo viento es caricia. Hablar de crisis es promoverla, y callar en la crisis es exaltar el conformismo. En vez de esto, trabajemos duro. Acabemos de una vez con la única crisis amenazadora, que es la tragedia de no querer luchar por superarla". ( Leturia, 2017)

Entonces, la crisis sanitaria del COVID 19, nos ha motivado a establecer nuevas formas de comunicación, atención al usuario y solucionar las situaciones críticas de cierre de brechas de conectividad de los estudiantes, especialmente de las comunidades campesinas y, como resultado se instaura un valor público con participación activa de servidores públicos, padres de familia y autoridades locales, donde se evidencien cambios en los servicios educativos.

A través del trabajo remoto, el 100\% de directores y docentes del ámbito jurisdiccional, atienden con la estrategia de APRENDO EN CASA a estudiantes que poseen conectividad de radio, televisión e internet, previo acompañamiento personalizado de la familia y reforzados por la educación comunitaria: ayni (colaboración mutua), aylluntin kuyanakuy (cultivar el amor familiar) y otros. Acción cumplida disciplinadamente, ya que no se cuenta con fallecimiento de docente ni padre de familia alguno, a pesar que más del 90\% de docentes habitan en Ica y Lima.

Para cerrar la brecha del $6 \%$ de estudiantes que no tienen acceso a los medios de comunicación referidos, por iniciativa del AGP y apoyo de AGI y AGA, se implementan la elaboración y entrega personalizada de GUÍAS DE APRENDIZAJE, por cada ciclo de estudios de EBR y EBA; documentos físicos (cartillas a colores) que orientan el buen entendimiento de los textos de lectura de matemática, comunicación, ciencia y tecnología, otorgadas por el MINEDU. Acción emprendida con el trabajo intelectual de especialistas en educación y aporte económico del 100\% de las municipalidades distritales y la Sub Gerencia de Castrovirreyna y, para su continuidad, con el convenio con el programa PAIS, asignación presupuestal del PP 090 y monitoreo de campo; porque "la Educación en Castrovirreyna no puede parar".

También, en comunidades dispersas desde 1950 hasta $5000 \mathrm{msnm}$ de altitud, se instalaron 8 estaciones de internet satelital, y la firma del convenio interinstitucional para la operación y cogestión del funcionamiento de una radio emisora entre la UGEL CASTROVIRREYNA con RADIO CASTROVIRREYNA, que dentro de 6 meses contará con la interconexión radial a nivel provincial.

En suma, las propuestas para enfrentar a la crisis del COVID 19, nace por la gestión colegiada primigenia de servidores públicos $\mathrm{y}$, compromiso de padres de familia $\mathrm{y}$

Implementando una gobernanza pública en la UGEL de Castrovirreyna - Riveros Poma,

Rómulo

DOI: https://doi.org/10.18800/cige2020.005 
Congreso Internacional de Gestión Educativa, 06 y 07 de noviembre de 2020.

Pontificia Universidad Católica del Perú

autoridades locales, implementándose una nueva gobernanza pública en educación, donde se enfatiza el rol de los ciudadanos en la formulación de las políticas públicas y la coproducción de servicios: enmarcado en la transparencia y lucha contra la corrupción.

\section{Referentes teóricos}

\section{Apunte histórico}

La realidad paupérrima social, cultural y económica de las Comunidades Campesinas de Huancavelica, muestran una eterna desatención de los gobernantes de turno a través de la historia. Por el cual, existe una situación motivada por la presencia de un amplio estrato social dominado y, en consecuencia, oprimido y deprimido, resultado de su peculiar proceso histórico... Por la índole de la colonización, por el medio vertical de difícil accesibilidad y comunicación... este estrato social mantiene una estructura que corresponde a una cultura de la pobreza, cultura dominada e integrada en forma particular en la sociedad nacional”. (Fuenzalida, Mayer, Escobar, Bourricaud, \& Matos Mar, 1970).

Reflejo colonial que se ha enraizado con el pensamiento republicano de Deústua, quien refería a los pobladores de contextos rurales: ¿Qué influencia podrá tener sobre esos seres, que sólo poseen la forma humana, las escuelas primarias más elementales? ¿Para qué aprenderán a leer, escribir y contar, la geografía y la historia y tantas otras cosas, los que no son personas todavía, los que no saben vivir como personas, los que no han llegado a establecer una diferencia profunda con los animales, ni tener ese sentimiento de dignidad humana principio de toda cultura? ¿Por qué habrían de ser más felices, con esas ideas, que los más no podrán aplicar en su vida extraña a la civilización y de que algunos podrían hacer uso contra sus semejantes? Solo un concepto intelectualista de la civilización puede concebir la felicidad en esas condiciones. (Bermejo \& Maquera, 2019).

Este pensamiento inhumano, fue base para la implementación de políticas de gobierno nacional, basadas en el centralismo limeño, las que se evidencian en nuestros días, con una pésima implementación de la política de salud y educación, sin servicios básicos. Situación social que se demostró objetivamente con la presencia de la crisis sanitaria del COVID 19; sin embargo, se dictaminan dispositivos legales de protección e higiene para "todos" los habitantes, de contextos urbanos y rurales, a pesar que los segundos, no cuentan mínimamente con instalación de agua potable, sino solamente sobreviven con agua entubada u puquiales naturales.

\section{Realidad en el sector Educación}

La pandemia por COVID-19 hizo que los sistemas educativos de todo el mundo se adapten a una condición que la brecha generacional exigía, es decir, trasladar las escuelas al ciberespacio de manera acelerada, sin importar la condición socioeconómica, geográfica o cultural, hecho que significa un cambio en la gestión educativa sus planes y programas. (Pérez \& Tufiño, 2020)

Como efecto de la presencia de casos del COVID 19, en el sector educación de Perú, se emite la RM No 160-2020-MINEDU, para el inicio del año escolar a través de la

Implementando una gobernanza pública en la UGEL de Castrovirreyna - Riveros Poma,

Rómulo

DOI: https://doi.org/10.18800/cige2020.005 
Congreso Internacional de Gestión Educativa, 06 y 07 de noviembre de 2020.

Pontificia Universidad Católica del Perú

implementación de la estrategia denominada "APRENDO EN CASA", a partir del 6 de abril de 2020 como medida del Ministerio de Educación para garantizar el servicio educativo mediante su prestación a distancia en las instituciones educativas públicas de Educación Básica, a nivel nacional, en el marco de la emergencia sanitaria para la prevención y control del COVID-19. (Diario El Peruano, 2020). Pero, en el $46 \%$ de Instituciones Educativas de EBR, EBA Y ETP de la UGEL Castrovirreyna, que están ubicados en contextos rurales, no se pudo implementar esta estrategia de aprendizaje, debido a que no cuentan con conectividad de radio, televisión e internet; incluso existen comunidades que no cuentan con la instalación de energía eléctrica. Desde ahí, se están implementando diferentes estrategias para cerrar esta brecha, para atender a estudiantes de hogares que viven en la exclusión social. Porque tener o no tener acceso a conexión y dispositivos tecnológicos, así mismo; es preciso analizar también la distribución y desigualdad según el nivel socioeconómico de los actores desde distintas perspectivas ya sea, ubicación geográfica, tipo de institución educativa entre otras (Pérez \& Tufiño, 2020)

Otra preocupación durante la gestión, se contrasta que algunos servidores del área administrativa de la UGEL Castrovirreyna, mantienen vigente el trabajo por "funciones", desestimando el mejoramiento de los aprendizajes de los estudiantes; a pesar que se debe asesorar la gestión pedagógica y administrativa de las instituciones educativas bajo su jurisdicción, fortaleciendo su autonomía institucional. (Diario El Peruano, 2003). Sin embargo, se implementan acciones de sensibilización para entender que ésta alcance una gestión bajo la forma de gobernanza, donde se imponen cambios en cuatro direcciones: (i) Favorecer la participación y articulación de distintos actores (docentes, directores, padres de familia, municipio entre otros) en el proceso de diseño e implementación de proyectos; (ii) Generar nuevas formas de organización para la gestión que conduzcan a un sistema de relaciones menos jerárquico y más horizontal entre los actores; (iii) Revitalizar, en el proceso de toma de decisiones, el funcionamiento de órganos existentes como COPALE, mesas o comisiones especiales; (iv) Impulsar la construcción de redes entre los actores de la comunidad escolar y de la comunidad local, todo con el objetivo de generar condiciones para una acción colectiva en torno a la transformación de las escuelas. (Ríos Céspedes, 2020)

Además, el Sector Educación está cumpliendo disciplinadamente la recomendación de las autoridades del Sector Salud "QUÉDATE EN CASA", como estrategia de prevención y salvaguardar la vida de las personas e intentar disminuir la posibilidad de contagios, ha implicado para el sector educativo desplegar acciones conducentes a continuar con el ciclo escolar vigente, no sin tensiones, conflictos y dificultades para los involucrados, dado el cambio de los roles de los padres de familia o figura parentales, a quienes esta contingencia les ha llevado a asumir un papel activo como co-educadores. A los docentes un trabajo monumental para impartir contenidos de acuerdo con el currículo escolar, revisión de trabajos de los estudiantes, incorporar estrategias para suscitar experiencias de aprendizaje en los alumnos a través de diversos medios y modalidades. (Hernández, 2020).

\section{Necesidad de una gestión pública}

Implementando una gobernanza pública en la UGEL de Castrovirreyna - Riveros Poma, Rómulo

DOI: https://doi.org/10.18800/cige2020.005 
Congreso Internacional de Gestión Educativa, 06 y 07 de noviembre de 2020.

Pontificia Universidad Católica del Perú

La finalidad de Ley $N^{\circ}$ 30057, Ley de Servicio Civil, es que las entidades públicas del Estado alcancen mayores niveles de eficacia y eficiencia, y presten efectivamente servicios de calidad a través de un mejor Servicio Civil, así como promover el desarrollo de las personas que lo integran. Pero, "todo es una literatura", porque en nuestro contexto convivimos con una limitada implementación de la Administración Pública, por encontrarse desarticulada y sin capacidad de gestionar la voluntad de sus ciudadanos, atado a intereses de los gestores públicos, los políticos y a grupos de interés ajenos a la sociedad civil.

Esta realidad motiva a implementar la transición de la administración a la nueva gestión pública, entre las causas que detonan esta transformación están precisamente las tres dimensiones que consideramos pilares del nuevo modelo de gestión pública... aquél que en el conjunto de redes de agencias funcione bien, es decir con eficiencia en el ejercicio de los fondos públicos, con eficacia en la interacción de agentes y agencias externas, y con legitimidad en la prestación del bien o servicio que produce", traducido esto último en la satisfacción del ciudadano y con rendición de cuentas. Estos tres elementos forman a su vez las características del modelo de gobernabilidad. (Díaz de León, 2013).

\section{Hacia una gobernanza educativa pública}

La gerencia en educación en sus distintos niveles, incluida la fase de diseño y concepción de las políticas públicas educacionales, hasta la gerencia de aula, al parecer ha perdido su horizonte axiológico más valioso como es el cultivo del afecto entre los individuos, que es lo trascendental... Sin embargo, la perspectiva sistémica debe mostrar que, entre todo gerente, gerencia del capital humano, docentes, estudiantes y contexto, existen múltiples relaciones que definen una interdependencia, donde cada actor participa en las funciones que le corresponde en el marco de un proceso educativo, creativo, emancipador y transformador. Esta visión integradora, debe estar presente en los llamados al diseño y concepción de las políticas públicas de una sociedad determinada. (Tovar Gómez, 2020).

\section{Desarrollo de la experiencia}

\section{Cierre de brechas de APRENDO EN CASA}

La producción de GUÍAS DE APRENDIZAJE, tiene la finalidad de promover el aprendizaje autónomo de estudiantes de EBR y EBA que no tienen conectividad de medios tecnológicos, es una iniciativa interna, diseñada y contextualizada por el equipo de especialistas de Educación de la UGEL Castrovirreyna. Material Educativo que se confeccionaron gracias al aporte económico de 12 Alcaldes de Municipalidades Distritales y el Gerente Subregional de Castrovirreyna, especialmente en la impresión a colores y la distribución a cada estudiante, en forma presencial, a pesar de la lejanía donde habitan. Acción cristalizada gracias a los permanentes acuerdos interinstitucionales.

La instalación de internet satelital, se realizaron en Comunidades Campesinas alejadas: Capillas, Chancahuasi, Chocoró, Colcabamba, Pauranga y Cocha. Además, en los distritos de Ticrapo, Huachos y Arma; estos 3 últimos por sus municipios distritales.

Implementando una gobernanza pública en la UGEL de Castrovirreyna - Riveros Poma, Rómulo

DOI: https://doi.org/10.18800/cige2020.005 
Congreso Internacional de Gestión Educativa, 06 y 07 de noviembre de 2020.

Pontificia Universidad Católica del Perú

Como la radio es un medio de comunicación masivo de mayor impacto en contextos rurales, en el mes de Setiembre de 2020, se implementó una RADIO EMISORA CASTROVIRREYNA, en los ambientes de la UGEL Castrovirreyna, previo convenio con un empresario de radiodifusión. Por este medio ya se difunden los programas de APRENDO EN CASA, microprogramas educativos y programas difundidas por trabajadores de nuestra entidad. Proyecto que nace gracias a una decisión colegiada de jefes de Línea, cuya meta es implementar una intercomunicación radial al 100\% de los 12 distritos de la jurisdicción administrativa.

Todos los procesos de acción de personal se realizan en forma virtual, incluido las notificaciones de sus resoluciones directorales (contrata, reasignación u otro) de docentes y trabajadores administrativos.

\section{Estableciendo una gestión pública}

Durante la crisis sanitaria del COVID 19, en cumplimiento del trabajo remoto, todos los trabajadores atienden a usuarios, también en forma remota, donde consignan en un aplicativo implementado en DRIVE en forma diaria, el resultado o actividad cumplida. Incluso, durante el trabajo presencial, cada trabajador deberá consignar su trabajo cumplido del día, en el mencionado archivo digital. Además, se aprobó con resolución directoral, la emisión de documentos internos y externos utilizando las firmas digitales.

\section{Lecciones aprendidas}

En la Unidad de Gestión Educativa Local de Castrovirreyna, se está dando los primeros pasos de la gestión por procesos, gracias a la participación dinámica de sus trabajadores, quienes están sensibilizándose para el cambio, gracias a la exigencia de la crisis sanitaria mundial.

Las coordinaciones interinstitucionales siempre tienen resultados satisfactorios, porque contribuyeron al cierre de brechas a estudiantes sin conectividad tecnológica y, en el mes de noviembre se podrá llegar al $100 \%$ de estudiantes con las GUIAS DE APRENDIZAJE, que es un modelo en la Región de Huancavelica, para atender a estudiantes sin conectividad.

La toma de decisiones en forma colegiada, siempre está orientado al fortalecimiento institucional.

\section{Agradecimientos}

Un agradecimiento cordial al equipo dinámico del Área de Gestión Pedagógica, Área de Gestión Institucional, Área de Gestión Administrativa y área de Asesoría Jurídica de la Unidad de Gestión Educativa Local de Castrovirreyna, quienes mostraron disponibilidad al cambio y emprender nuevos retos de gestión pública, en plena crisis del COVID 19.

Asimismo, es resaltar el aporte incondicional del Gerente Subregional de Castrovirreyna, a los Alcaldes de los distritos de: Arma, Capillas, Ticrapo,

Implementando una gobernanza pública en la UGEL de Castrovirreyna - Riveros Poma,

Rómulo

DOI: https://doi.org/10.18800/cige2020.005 
Congreso Internacional de Gestión Educativa, 06 y 07 de noviembre de 2020.

Pontificia Universidad Católica del Perú

Castrovirreyna, Aurahuá, Chupamarca, Cocas, Huachos, Huamatambo, Mollepampa, San Juan y Tantará.

\section{Referencias bibliográficas}

Leturia, M. (01 de Abril de 2017). Breves consideraciones sobre el acto de creación. Obtenido de Breves consideraciones sobre el acto de creación: http://sedici.unlp.edu.ar/bitstream/handle/10915/60498/Documento_completo.pdfPDFA.pdf?sequence $=1$ \&isAllowed $=\mathrm{y}$

Bermejo, S., \& Maquera, Y. (01 de Mayo de 2019). Interpretación de la escuela rural andina en. Educare de la Universidad Nacional Costa Rica, 32(2), 6. doi:http://dx.doi.org/10.15359/ree.23-2.4

Diario El Peruano. (29 de Julio de 2003). Ley general de Educacioón No 28044. Diario Oficial El Peruano(8437), pág. 14. Recuperado el 12 de setiembre de 2020, de https://www.unj.edu.pe/images/pdf/calidad/marconormativo/LEY\%2028044\%20LEY\%20 GENERAL\%20DE\%20EDUCACION.pdf

Diario El Peruano. (31 de Marzo de 2020). Normas Legales del diario El peruano. $R M N^{o} 160$ 2020-MINEDU, págs. 9 - 10. Recuperado el 13 de octubre de 2020

Díaz de León, C. (Marzo de 2013). Nueva Gestión Pública y Gobernanza: Desafíos en su Implementación. Obtenido de Nueva Gestión Pública y Gobernanza: Desafíos en su Implementación: https://d1wqtxts1xzle7.cloudfront.net/45661959/Nueva_Gestion_Publica_y_Gobernanza_Desafios_en_su_Implementacion.pdf?1463400622=\&response-contentdisposition=inline\%3B+filename\%3DNueva_Gestion_Publica_y_Gobernanza_Desaf.pdf\& Expires $=1604211582 \&$ Signature $=\mathrm{cM}$

Fuenzalida, F., Mayer, E., Escobar, G., Bourricaud, F., \& Matos Mar, J. (1970). El indio y el poder en el Perú. Lima: Moncloa-Campodónico.

Hernández, A. R. (20 de Mayo de 2020). COVID-19: el efecto en la gestión educativa. RELAIS, 3(1), 37-41. Obtenido de http://revistasinvestigacion.lasalle.mx/index.php/relais/article/view/2640/2592

Pérez, M., \& Tufiño, A. (18 de Mayo de 2020). Teleeducación y COVID-19. CienciAmérica, 9(2), 5. http://201.159.222.118/openjournal/index.php/uti/article/view/296/447

Ríos Céspedes, A. (20 de mayo de 2020). Gobernabilidad y gobernanza en las Unidades de Gestión Educativa Local del Perú: Una mirada desde el conocimiento de sus funciones. (R. I. Educación, Ed.) doi:https://doi.org/10.35362/rie8313832

Tovar Gómez, F. (19 de enero de 2020). http://www.grupocieg.org/archivos_revista/Ed.43(5361)-Tovar\%20Francisco_articulo_id617.pdf. Obtenido de http://www.grupocieg.org/archivos_revista/Ed.43(53-61)Tovar\%20Francisco_articulo_id617.pdf: http://www.grupocieg.org/archivos_revista/Ed.43(53-61)Tovar\%20Francisco_articulo_id617.pdf

Implementando una gobernanza pública en la UGEL de Castrovirreyna - Riveros Poma, Rómulo

DOI: https://doi.org/10.18800/cige2020.005 